\title{
IMPACTO DA LUZ, pH, ÁCIDO ASCÓRBICO E GLICOSE NA ESTABILIDADE DE ANTOCIANINAS DA FONTE NÃO USUAL "MARIA-PRETINHA" (Solanum americanum, Mill.)
}

\author{
ALINE MANKE NACHTIGALL* \\ POLLYANNA IBRAHIM SILVA** \\ MICHELE CORRÊA BERTOLDI** \\ PAULO CÉSAR STRINGHETA***
}

\begin{abstract}
O fruto de maria-pretinha (Solanum americanum) consiste em fonte não usual, porém viável de produção de antocianinas. Sendo assim, objetivouse avaliar a estabilidade do extrato antociânico obtido dessa fonte vegetal, após extração com metanol acidificado $(0,05 \% \mathrm{HCl})$, frente a fatores degradativos como luz, pH, ácido ascórbico e glicose. Sua estabilidade foi estimada determinando-se os valores de absorbância no comprimento de onda de máxima absorção em função do tempo, mediante os quais foram calculadas as constantes de velocidade de degradação $(k)$ e o tempo de meiavida $\left(t_{1 / 2}\right)$ de cada sistema. Os efeitos da luz e da concentração de 400 ppm de ácido ascórbico foram os mais destrutivos para a cor do pigmento, especialmente em pH 4,0. A adição de ácido ascórbico acelerou a degradação do pigmento antociânico. A glicose não afetou a estabilidade das antocianinas. Os pigmentos apresentaram maior estabilidade em pH 2,0. A interação negativa de ácido ascórbico e antocianinas merece mais investigações, já que a aplicação de pigmentos antociânicos na indústria de alimentos, especialmente em sucos e refrigerantes, mostrouse bastante promissora.
\end{abstract}

PALAVRAS-CHAVE: ANTOCIANINAS - ESTABILIDADE; DEGRADAÇÃO; Solanum americanum, Mill. 


\section{INTRODUÇÃO}

A busca por matérias-primas para a produção de corantes naturais com propriedades adequadas ao uso na indústria de alimentos processados tem levado pesquisadores a investigar praticamente toda a biosfera. A maria-pretinha (Solanum americanum), erva cosmopolita que nasce espontaneamente, apresenta fácil cultivo em solos pobres, com produção considerável de pequenos frutos pretos por planta. No Brasil, aparece desde o Nordeste até o Sul, incluindo os estados do Centro-Oeste. É frequente em lavouras anuais, lavouras perenes, pomares, jardins, terrenos baldios, campos e pastagens. Embora considerados tóxicos enquanto verdes, os frutos maduros são comestíveis e ricos em antocianinas (CARIBÉ e CAMPOS, 1991; SILVA, 1996).

As antocianinas, pertencentes à classe dos flavonóides, são responsáveis pela coloração atrativa de flores e frutos, e reconhecidas como fontes alternativas aos corantes sintéticos vermelhos. Além dos atributos de cor, o interesse por esses compostos tem se intensificado em função de seus efeitos benéficos à saúde (COOPER-DRIVER, 2001; CONSTANT, STRINGHETA e SANDI, 2002; DEL POZO-INSFRAN, PERCIVAL e TALCOTT, 2006; HOGAN et al., 2010). Entretanto, existem muitas limitações para sua aplicação comercial em alimentos devido ao alto custo e instabilidade. A estabilidade das antocianinas depende de sua estrutura química, temperatura, $\mathrm{pH}$, concentração, tipo de solvente, oxigênio, luz, estrutura do pigmento e a presença de substâncias capazes de reagir reversível ou irreversivelmente com esses flavonóides, entre outras (CONSTANT, STRINGHETA e SANDI, 2002; BRENES, DEL POZO-INSFRAN e TALCOTT, 2005; DE ROSSO e MERCADANTE, 2007; MOLLOV et al., 2007; SADILOVA et al., 2009). Sabe-se que compostos naturalmente presentes nos alimentos, como açúcares e ácido ascórbico, podem influenciar a estabilidade das antocianinas. Vários estudos analisando a influência desses compostos são encontrados na literatura, entretanto, afirmações contraditórias levam a resultados não conclusivos (TSAI, HSIEH e HUANG, 2004; RABABAH, EREIFEJ e HOWARD, 2005).

Além da instabilidade, há escassez de antocianinas no mercado por falta de fontes e técnicas de produção adequadas e economicamente viáveis (CONSTANT, STRINGHETAe SANDI, 2002). Esses obstáculos, aliados à percepção do consumidor de que pigmentos naturais são mais saudáveis, estão gerando vários estudos (FALCÃO et al., 2003, LIMA, MÉLO e LIMA, 2005; MOLLOV et al., 2007; SADILOVA et al., 2009). Uma das alternativas frente aos problemas relacionados ao uso de antocianinas envolve a identificação de novas fontes produtoras mais estáveis, além da aplicação de técnicas que venham a contribuir para a melhoria da estabilidade desses pigmentos (CONSTANT, 2003; BRENES, DEL POZO-INSFRAN e TALCOTT, 2005; MONTES et al., 2005; MOLLOV et al., 2007).

SILVA (1996), ao determinar os teores de antocianinas em frutos de maria-pretinha, encontrou resultados variando de $150-200 \mathrm{mg} .100 \mathrm{~g} \mathrm{~g}^{-1}$. Tais teores se aproximam dos de outras fontes de antocianinas, como açaí (111 mg.100 g-1) (RUFINO et al., 2010), capim-gordura (99 mg.100 g $\mathrm{g}^{-1}$ ) (TEIXEIRA, STRINGHETA e OLIVEIRA, 2008) e repolho roxo $\left(175 \mathrm{mg}^{1} 100 \mathrm{~g}^{-1}\right)$ (LOPES, QUADRI e QUADRI, 2006), demonstrando o potencial da utilização do fruto como fonte de antocianinas. Levandose em conta a busca crescente por novas fontes comerciais de corantes naturais e a facilidade de propagação da planta, a produção de maria-pretinha em escala comercial deveria ser incentivada, especialmente em regiões desfavorecidas pelas características do solo.

Tendo-se em vista a tendência mundial e irreversível do uso crescente de corantes naturais, bem como o desuso dos corantes sintéticos, procurou-se por meio deste trabalho levantar dados sobre a estabilidade de pigmentos antociânicos extraídos de fonte não usual de antocianinas, a maria-pretinha.

\section{MATERIAL E MÉTODOS}

Os frutos de maria-pretinha foram colhidos no campo experimental do Departamento de Fitotecnia, da Universidade Federal de Viçosa, Viçosa (MG), sendo as antocianinas extraídas com 
metanol acidificado $(0,05 \% \mathrm{HCl})$ de acordo com os procedimentos descritos por FULEKI e FRANCIS (1968). O extrato concentrado obtido foi armazenado na ausência de luz e baixa temperatura $\left(5^{\circ} \mathrm{C}\right)$ para análises posteriores.

Nas condições adotadas neste estudo levou-se em conta o comportamento das antocianinas frente a diferentes valores de $\mathrm{pH}$ (tampão fosfato/citrato $\mathrm{pH}$ 2,0; 3,0 e 4,0), ausência e presença de luz (40 W, 2.500 lux), concentração de ácido ascórbico (100ppm, 200 ppm e 400 ppm, na ausência de luz) e concentração de glicose $(2 \%, 4 \%$ e $6 \%$, em presença e ausência de luz). Os procedimentos seguiram a metodologia descrita por STRINGHETA (1991), com algumas modificações.

As leituras de absorbância foram realizadas em quintuplicata a cada 24 horas, no comprimento de onda de máxima absorção, obtido mediante varredura do espectro de absorção (390 a $600 \mathrm{~nm}$ ), usando-se espectrofotômetro GBC UV-Visível 911A (Melbourne, Austrália). Mediu-se a absorbância diariamente até leitura próxima a 0,2 , quando então os tubos foram descartados.

Os cálculos da constante de velocidade de degradação e do tempo de meia-vida foram obtidos pelos dados de absorbância versus tempo, assumindo-se reação de primeira-ordem:

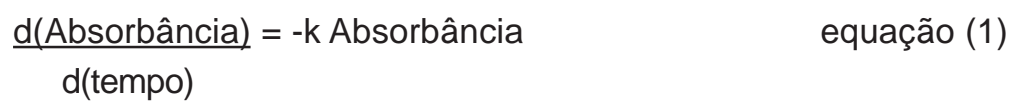

Estimou-se a constante da reação mediante regressão linear do logaritmo neperiano da absorbância (In Absorbância) versus tempo. Para o cálculo do tempo de meia-vida utilizou-se a relação:

$$
\frac{0,693}{\mathrm{k}}=\mathrm{t}_{1 / 2} \quad \text { equação (2) }
$$

\section{RESULTADOS E DISCUSSÃO}

Ao observar a Figura 1 e a Tabela 1 percebe-se que o pH exerce grande influência sobre a estabilidade dos pigmentos antociânicos, tanto na presença como na ausência de luz, e que o pigmento é mais estável em pH mais ácido (maior estabilidade observada em $\mathrm{pH}$ 2,0).

Segundo STRINGHETA (1991), a taxa de degradação de antocianinas é profundamente afetada pelo $\mathrm{pH}$ e o aumento da acidez favorece a estabilidade do pigmento. Sabe-se que as antocianinas apresentam coloração vermelho intensa em pH abaixo de 3. Aumentando-se o pH da solução, essa coloração tende a desaparecer tornando as soluções incolores na faixa de pH entre 4 e 5 . Em meio bastante ácido, as antocianinas encontram-se quase totalmente na forma catiônica (cátion flavilium), sua forma mais importante e também a mais estável (FRANCIS, 1982; STRINGHETA, 1991). Com o aumento do $\mathrm{pH}$ ocorre a desprotonação do cátion flavilium, originando as formas carbinol e chalcona com a consequente descoloração do pigmento.

O comportamento mostrado na Figura 1 e na Tabela 1 representa grande preocupação para a indústria. A maioria dos alimentos apresenta $\mathrm{pH}$ maior que 4, condição em que o pigmento encontrase bastante instável, perdendo rapidamente sua coloração. Para contornar os problemas relacionados com o pH, técnicas de copigmentação são utilizadas para aumentar a estabilidade do pigmento, além de outras técnicas que impedem seu contato direto com o alimento como, por exemplo, o microencapsulamento (PERIN, 1999; FALCÃO et al., 2003). 


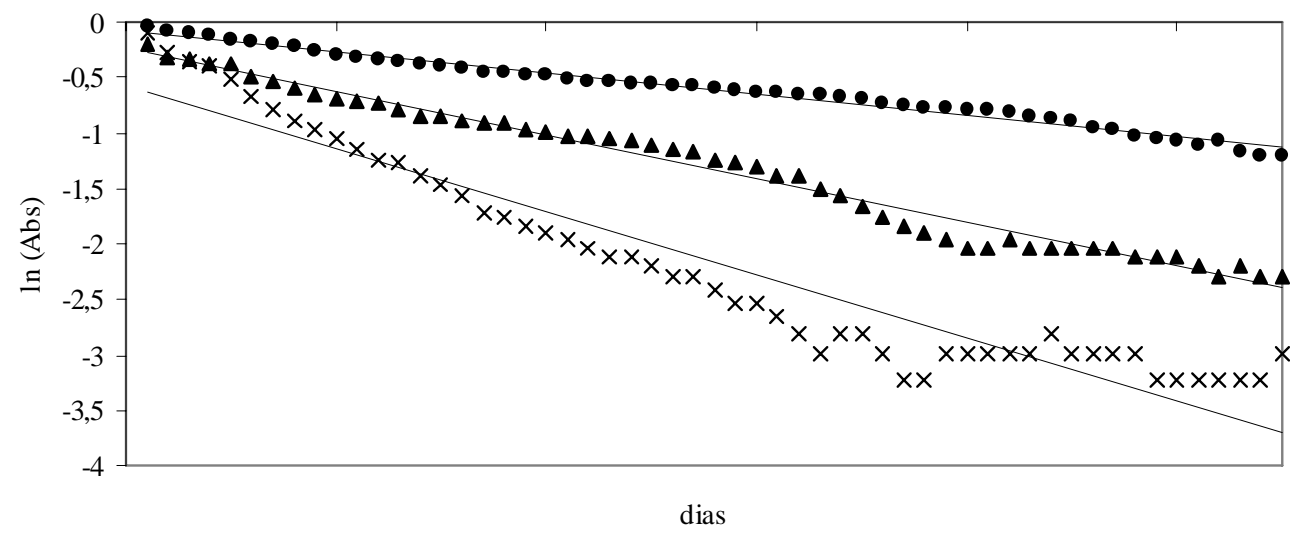

$\begin{array}{llllllllllll}\text { B } & 0 & 2 & 4 & 6 & 8 & 10 & 12 & 14 & 16 & 18 & 20\end{array}$

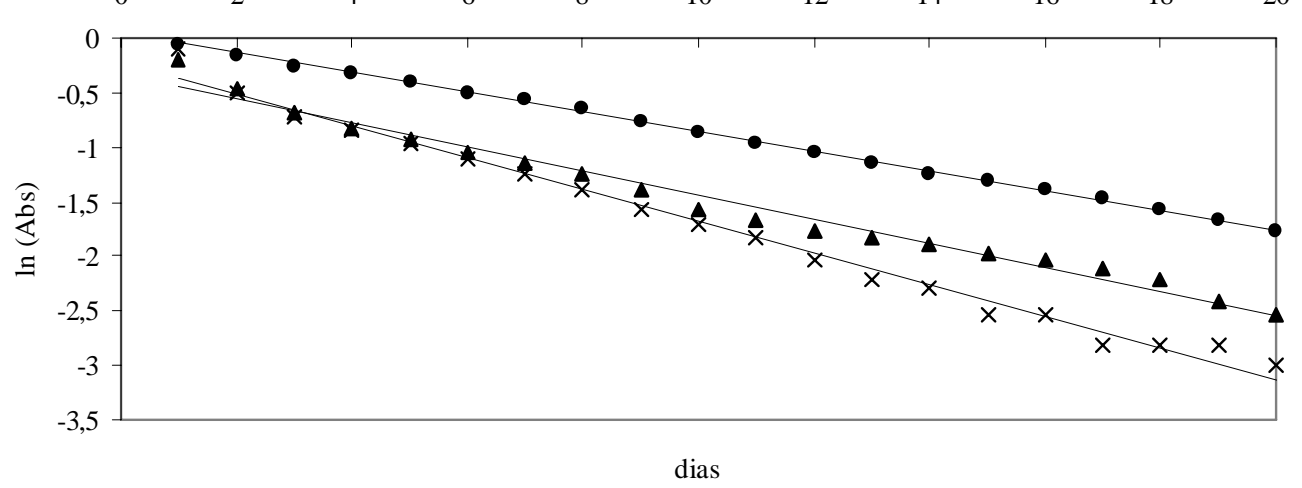

$\bullet \mathrm{pH}=2,0 \Delta \mathrm{pH}=3,0 \times \mathrm{pH}=4,0$

\section{FIGURA 1 - LOGARITMO NEPERIANO DA ABSORBÂNCIA DO EXTRATO ANTOCIÂNICO DE MARIA-PRETINHA EM SISTEMAS-TAMPÃO NA AUSÊNCIA (A) E PRESENÇA DE LUZ (B)}

STRINGHETA (1991), ao estudar a estabilidade de pigmentos da inflorescência de capimgordura, observou que a luz afetou profundamente a estabilidade da cor de extratos brutos e parcialmente a de extratos purificados de antocianinas a pH 2. DE ROSSO e MERCADANTE (2007) também observaram efeito prejudicial da luz em antocianinas de acerola e açaí, quando comparadas com os mesmos pigmentos na ausência de luz.

TABELA 1 - VALORES OBTIDOS PARA CONSTANTE DE VELOCIDADE DE REAÇÃO (k) E TEMPO DE MEIA-VIDA EM DIAS $\left(T_{1 / 2}\right)$ DO EXTRATO ANTOCIÂNICO EM SISTEMAS-TAMPÃO NA PRESENÇA E AUSÊNCIA DE LUZ

\begin{tabular}{lcccc}
\hline Condições & $\mathbf{p H}$ & $\mathbf{k}$ & $\mathbf{t}_{\mathbf{1 / 2}}$ & $\mathbf{R}^{\mathbf{2}}$ \\
\hline Presença de & 2,0 & 0,0901 & 7,69 & 0,9986 \\
luz & 3,0 & 0,1106 & 6,26 & 0,9842 \\
& 4,0 & 0,1451 & 4,78 & 0,9889 \\
\hline \multirow{2}{*}{ Ausência de } & 2,0 & 0,0193 & 35,91 & 0,9840 \\
luz & 3,0 & 0,0396 & 17,5 & 0,9817 \\
& 4,0 & 0,0570 & 12,16 & 0,9096 \\
\hline
\end{tabular}


Reduzida estabilidade à luz impede que o produto alimentício seja exposto aos olhos do consumidor em embalagens transparentes. Nesse sentido, estudos devem ser implementados com o objetivo de buscar técnicas que possam minimizar o efeito da luz sobre as antocianinas.

A equação de regressão linear do logaritmo neperiano da absorbância versus tempo ajustouse adequadamente aos dados obtidos no experimento referente ao efeito da luz, confirmando que a degradação do pigmento realmente é representada por reação de primeira-ordem, já que os valores de $\mathrm{R}^{2}$ ficaram bastante próximos a 1,0 (Tabela 2).

TABELA 2 - EQUAÇÃO DE REGRESSÃO LINEAR DOS VALORES DE $k$ EM FUNÇÃO DO pH PARA A FAIXA DE 2,0 A 4,0 E OS RESPECTIVOS VALORES DE ${ }^{2}$

\begin{tabular}{ccc}
\hline Fator & Equação de Regressão Linear & $\mathbf{R}^{2}$ \\
\hline LuZ & $Y=0,0275 x+0,0603$ & 0,9789 \\
EScuro & $Y=0,0189 x+0,0009$ & 0,9980 \\
\hline
\end{tabular}

Observa-se pela Tabela 2 que os valores de absorbância obtidos para a degradação do pigmento no escuro ajustaram-se melhor ao modelo de cinética de primeira-ordem, comparados aos dados encontrados para o mesmo experimento na presença de luz. Isto pode ser atribuído ao fato da degradação do pigmento no escuro ser mais lenta e assim apresentar melhor linearidade.

Ao avaliar a inclinação da reta obtida na equação de regressão linear percebe-se que a luz apresentou acentuado efeito na perda do pigmento antociânico. Obteve-se inclinação de 0,0275 na presença de luz e de 0,0189 na ausência de luz, indicando maior degradação do pigmento quando o fator luz está presente. Esses resultados estão de acordo com a literatura, que relata amplamente o efeito deletério da luz sobre pigmentos antociânicos (CONSTANT, 2003; LIMA, MÉLO e LIMA, 2005; PROVENZI et al., 2006).

Com relação ao emprego de ácido ascórbico, verificou-se relação inversa entre o aumento da concentração do ácido e a estabilidade das antocianinas (Figura 2).

Por analogia com o experimento anterior, e analisando os valores de $\mathrm{R}^{2}$ apresentados na Tabela 3, pode-se inferir que as equações obtidas representam adequadamente os dados do presente estudo e que a equação de primeira-ordem simula melhor a reação de degradação do pigmento antociânico de frutos de maria-pretinha frente ao ácido ascórbico.

\section{TABELA 3 - VALORES OBTIDOS PARA CONSTANTE DE VELOCIDADE DE DEGRADAÇÃO DO PIGMENTO (k) E TEMPO DE MEIA-VIDA EM DIAS $\left(T_{1 / 2}\right)$ DO EXTRATO ANTOCIÂNICO COM 100, 200 E 400 ppm DE ÁCIDO ASCÓRBICO}

\begin{tabular}{lccccccccc}
\hline & \multicolumn{3}{c}{ 100 ppm } & \multicolumn{2}{c}{ 200 ppm } & \multicolumn{3}{c}{ 400 ppm } \\
\hline $\mathbf{p H}$ & $\mathbf{k}$ & $\mathbf{R}^{2}$ & $\mathbf{T}_{\mathbf{1} / 2}$ & $\mathbf{k}$ & $\mathbf{R}^{2}$ & $\mathbf{t}_{\mathbf{1} / 2}$ & $\mathbf{k}$ & $\mathbf{R}^{2}$ & $\mathbf{t}_{\mathbf{1 / 2}}$ \\
\hline 2,0 & 0,0532 & 0,9394 & 13,0 & 0,0727 & 0,9622 & 9,5 & 0,0988 & 0,9476 & 7,0 \\
3,0 & 0,0547 & 0,9782 & 12,7 & 0,0815 & 0,9743 & 8,5 & 0,1281 & 0,9831 & 5,4 \\
4,0 & 0,0767 & 0,9467 & 9,0 & 0,1063 & 0,9567 & 6,5 & 0,1521 & 0,9399 & 4,5 \\
\end{tabular}



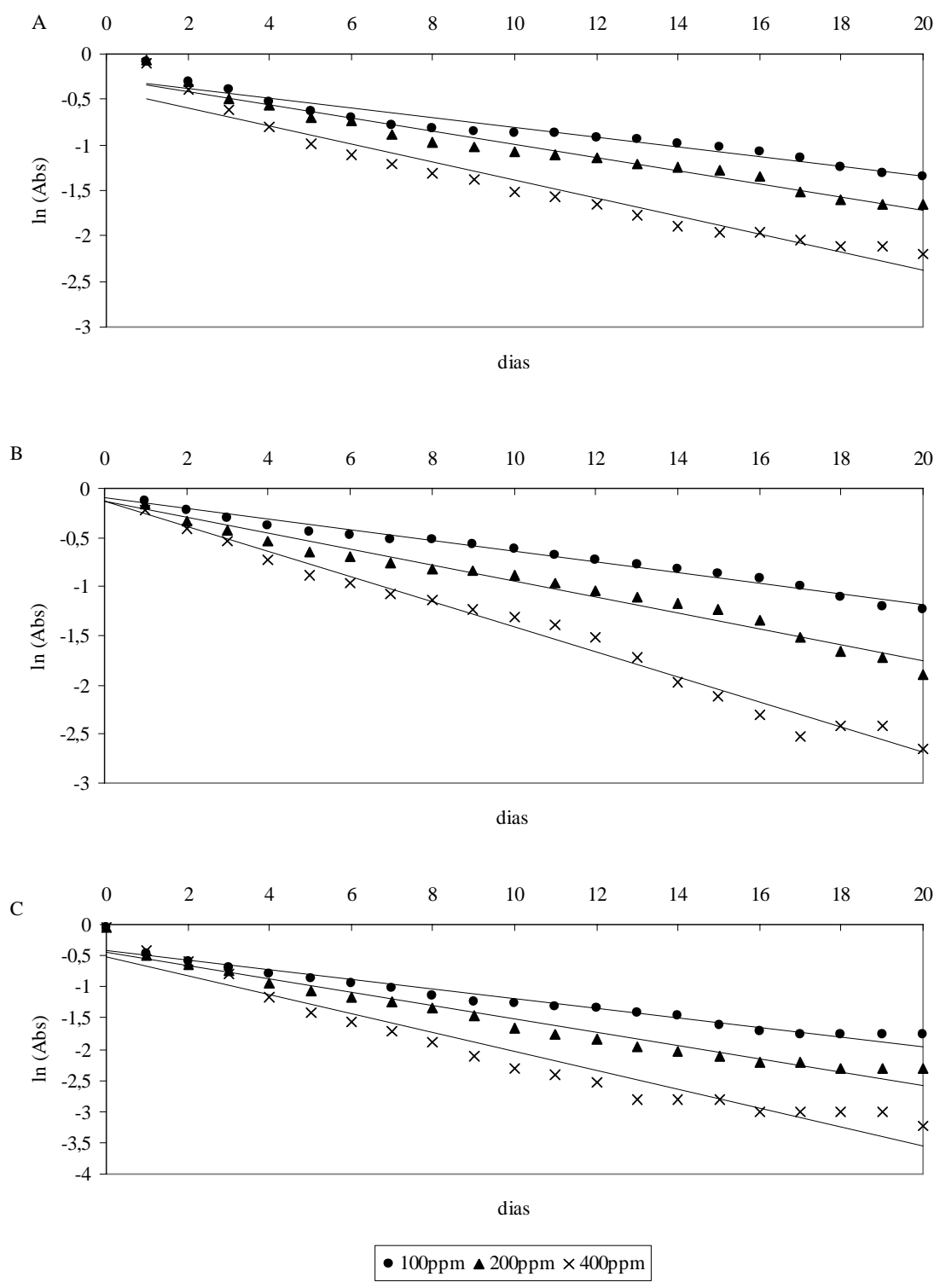

\section{FIGURA 2 - LOGARITMO NEPERIANO DA ABSORBÂNCIA DO EXTRATO ANTOCIÂNICO DE MARIA-PRETINHA ACRESCIDOS DE DIFERENTES CONCENTRAÇÕES DE ÁCIDO ASCÓRBICO EM pH 2,0 (A), 3,0 (B) E 4,0 (C)}

Analisando a Tabela 3 verifica-se dependência entre as constantes de degradação e os valores de $\mathrm{pH}$. Para $\mathrm{pH}$ 2,0 e 3,0 a diferença foi proporcionalmente menor que para o $\mathrm{pH}$ 4,0, confirmando $\mathrm{o}$ efeito altamente destrutivo do aumento de $\mathrm{pH}$ sobre a retenção da cor do pigmento antociânico de frutos de maria-pretinha.

Comparando as constantes de velocidade de degradação do pigmento antociânico (k) e tempos de meia-vida ( $\left.\mathrm{t}_{1 / 2}\right)$ para o experimento com adição de ácido ascórbico (Tabela 3) com os resultados obtidos para o experimento na ausência de luz e sem adição de ácido ascórbico (Tabela 1), as constantes de velocidade de degradação do pigmento na presença do ácido ascórbico foram sempre superiores. Percebe-se, ainda, que a degradação é proporcional ao aumento da concentração de ácido ascórbico. Esses dados vêm corroborar resultados obtidos por outros autores em que a presença de ácido ascórbico aumentou a taxa de degradação do pigmento (GARZÓN e WROLSTAD, 2002; BRENES, DEL POZO-INSFRAN e TALCOTT, 2005; DE ROSSO e MERCADANTE, 2007). 
DE ROSSO e MERCADANTE (2007), estudando a interação de ácido ascórbico com antocianinas, verificaram a degradação de ambos (decréscimo da cor e perda da qualidade nutricional). Esse fato é importante para produtos como sucos, que além da apresentação de cor agradável ao consumidor envolve o valor nutricional do alimento.

O mecanismo para a degradação de antocianinas na presença de ácido ascórbico, inicialmente proposto por JURD (1972) e reforçado por POEI-LANGSTON e WROLSTAD (1981), consiste em condensação direta do ácido ascórbico no carbono 4 da molécula de antocianina, causando a perda de ambos. Entretanto, de acordo com IACOBUCCI e SWEENY (1983), a perda da cor ocorre por causa da clivagem do anel pirilium por mecanismo envolvendo a produção de radicais livres com a participação do ácido ascórbico.

Resultados contraditórios foram obtidos por MORENO-ALVAREZ et al. (2002) ao observarem que a adição de ácido ascórbico ao suco de amora-preta (Rubus glaucus) aumentou a conservação do suco e proporcionou manutenção dos teores de antocianinas. De acordo com SADILOVA et al. (2009), a estrutura da antocianina (especialmente a glicosilação e o tipo de acilação), exerce influência na reatividade da molécula frente ao ácido ascórbico. Demonstraram, como exemplo, que malvidinadiglicosídeo é mais estável que seu monoglicosídeo. BOBBIO, BOBBIO e SOUZA (1987) identificaram duas antocianinas nos frutos de maria-pretinha, a malvidina-3-soforosídeo-5-glucosídeo e a malvidina3-(di-cafeoil-soforosídeo)-5-glucosídeo.

Há ainda estudos que não revelam efeitos significativos da presença do ácido ascórbico na cor e na estabilidade dos pigmentos (RABABAH, EREIFEJ e HOWARD, 2005).

Os dados referentes à constante de velocidade de degradação das antocianinas $(k)$ e o tempo de meia-vida $\left(t_{1 / 2}\right)$ frente à adição de glicose estão resumidos na Tabela 4. Observando os valores de $\mathrm{R}^{2}$ pode-se afirmar que os dados obtidos foram devidamente representados pelas curvas do logaritmo neperiano da absorbância versus tempo e que a reação de degradação do pigmento antociânico mais uma vez obedeceu a reação de primeira-ordem.

Ao avaliar os valores de $k$ e $t_{1 / 2}$ verificou-se relação direta entre a estabilidade, as variações de $\mathrm{pH}$ e a oferta de luz, assim como ocorreu para os ensaios anteriores. Os valores de $\mathrm{k}$ aumentaram com a elevação do $\mathrm{pH}$, indicando incremento da degradação do pigmento. O mesmo pode ser observado na ausência de luz, porém os valores de $\mathrm{k}$ foram inferiores aos obtidos na presença de luz em toda a faixa de $\mathrm{pH}$ estudada.

\section{TABELA 4 - VALORES OBTIDOS PARA CONSTANTE DE VELOCIDADE DE DEGRADAÇÃO DO PIGMENTO (k) E TEMPO DE MEIA-VIDA ( $T_{1 / 2}$ ), EM DIAS, DO EXTRATO ANTOCIÂNICO EM SISTEMAS-MODELO UTILIZANDO QUATRO CONCENTRAÇÕES DE GLICOSE}

\section{Presença de luz}

\begin{tabular}{|c|c|c|c|c|c|c|c|c|c|c|c|c|}
\hline \multirow{2}{*}{ pH } & \multicolumn{3}{|c|}{$0 \%$ de glicose } & \multicolumn{3}{|c|}{$2 \%$ de glicose } & \multicolumn{3}{|c|}{$4 \%$ de glicose } & \multicolumn{3}{|c|}{$6 \%$ de glicose } \\
\hline & $\mathbf{K}$ & $\mathbf{R}^{2}$ & $t_{1 / 2}$ & k & $\mathbf{R}^{2}$ & $T_{1 / 2}$ & $\mathrm{~K}$ & $\mathbf{R}^{2}$ & $t_{1 / 2}$ & $\mathbf{k}$ & $\mathbf{R}^{2}$ & $t_{1 / 2}$ \\
\hline 2,0 & 0,1943 & 0,994 & 3,6 & 0,1316 & 0,979 & 5,3 & 0,1449 & 0,989 & 4,8 & 0,1143 & 0,977 & 6,1 \\
\hline 3,0 & 0,3302 & 0,936 & 2,1 & 0,2685 & 0,907 & 2,6 & 0,2531 & 0,893 & 2,7 & 0,2175 & 0,899 & 3,2 \\
\hline 4,0 & 0,5426 & 0,897 & 1,3 & 0,5475 & 0,931 & 1,3 & 0,4862 & 0,914 & 1,4 & 0,4964 & 0,876 & 1,4 \\
\hline
\end{tabular}

Ausência de Luz

\begin{tabular}{lllllllllllll}
\hline 2,0 & 0,0176 & 0,982 & 39,4 & 0,0179 & 0,981 & 38,7 & 0,0172 & 0,989 & 40,3 & 0,0168 & 0,997 & 41,2 \\
3,0 & 0,0271 & 0,931 & 25,6 & 0,0241 & 0,924 & 28,7 & 0,0254 & 0,945 & 27,3 & 0,0492 & 0,978 & 14,1 \\
4,0 & 0,0512 & 0,911 & 13,5 & 0,0459 & 0,881 & 15,1 & 0,0464 & 0,863 & 14,9 & 0,0611 & 0,964 & 11,3 \\
\hline
\end{tabular}


Pela análise dos dados da Tabela 4, não houve grande variação na estabilidade do extrato antociânico de maria-pretinha em relação às diferentes concentrações de glicose. No entanto, vários trabalhos demonstraram efeito prejudicial da interação de açúcares na estabilidade das antocianinas (DYRBY, WESTGAARD e STAPELFELDT, 2001) e que esse efeito depende do tipo de açúcar (HUBBERMANN et al., 2006). Foi relatado que concentrações elevadas de açúcares (>20\%) causam decréscimo na atividade de água, o que leva ao aumento da estabilidade das antocianinas (TSAI, HSIEH e HUANG, 2004).

SADILOVA et al. (2009) afirmaram que houve melhora da estabilidade de antocianinas em sucos com adição de açúcares, a qual mostrou-se dependente do tipo de processamento a que os sucos foram submetidos, o que modifica, consequentemente, o meio em que o pigmento se encontra e a interação desse com compostos supostamente protetores. Entretanto, MALIEN-AUBERT, DANGLES e AMIOT (2001) observaram que a adição de açúcares não exerceu influência na estabilidade da coloração de antocianinas.

Já foi relatado que os produtos de degradação de açúcares formados durante o aquecimento, como o 5-hidroximetilfurfural, furfural e seus derivados são responsáveis pela perda de pigmentos (DEBICKY-POSPISIL et al., 1983; SADILOVA et al., 2009), provavelmente por reações de condensação com compostos presentes no meio.

Em experimentos utilizando altas concentrações de açúcares, normalmente recorre-se ao aquecimento para proporcionar total solubilização, ocorrendo a formação dos compostos de degradação, como o furfural e o hidroximetilfurfural. Ao se utilizar baixas concentrações de açúcar não há necessidade de aquecimento e o efeito prejudicial à coloração não é percebido. Desta forma, as concentrações de açúcar utilizadas no presente estudo não levaram à degradação do pigmento antociânico de maria-pretinha.

\section{CONCLUSÃO}

O efeito da luz foi o mais destrutivo para a cor das antocianinas de maria-pretinha, especialmente em pH 4,0. Os extratos em soluções com pH 2,0 apresentaram maior estabilidade comparados aos de $\mathrm{pH}$ 3,0 e 4,0. A adição de ácido ascórbico acelerou a degradação do pigmento antociânico. Os ensaios de estabilidade com diferentes concentrações de glicose não apresentaram influência considerável sobre a estabilidade do pigmento.

A interação negativa de ácido ascórbico e antocianinas merece mais investigações pela avaliação de fontes produtoras de pigmentos antociânicos mais estáveis, ou de técnicas de copigmentação que protejam a estrutura da antocianina contra a degradação, já que a aplicação de pigmentos antociânicos na indústria de alimentos, especialmente em sucos e refrigerantes, é bastante promissora.

\section{ABSTRACT \\ IMPACT OF LIGHT, pH, ASCORBIC ACID AND GLUCOSE ON ANTHOCYANIN STABILITY OF THE NON-USUAL SOURCE "MARIA-PRETINHA" (Solanum americanum, Mill.)}

The maria-pretinha fruit (Solanum americanum) is a non-usual, but feasible source to production of anthocyanins. Thus, the aim of this work was to evaluate the stability of anthocyanins obtained from these fruit, after extraction with acidified methanol $(0.05 \% \mathrm{HCl})$, under degrading factors such as light, $\mathrm{pH}$, addition of ascorbic acid and glucose. The stability was evaluated by the decrease of absorbance values along the time, measured in the maximum absorption wavelength of these pigments. It was possible to calculate the degradation rate constants $(k)$, as well as the half-life time $\left(t_{1 / 2}\right)$ to each system. The effects of light and addition of $400 \mathrm{ppm}$ ascorbic acid were more destructive to the pigment, mainly at $\mathrm{pH} 4.0$. Addition of ascorbic acid increased the degradation of anthocyanins. The presence of glucose didn't affect the stability of anthocyanins. Best pigment's stability condition was observed at $\mathrm{pH}$ 2.0. The negative interaction between ascorbic acid and anthocyanins deserve further investigations because the application of anthocyanin pigments in the food industry, specially in juice and sodas, is considerably promising. 


\section{REFERÊNCIAS}

1 BOBBIO, P.A.; BOBBIO, F.O.; SOUZA, S.C. Solanum americanum as a source of anthocyanins for use in foods. Acta Alimentaria, v.16, n.2, p.155-158, 1987.

2 BRENES, C.H.; DEL POZO-INSFRAN, D.; TALCOTT, S.T. Stability of copigmented anthocyanins and ascorbic acid in a grape juice model system. Journal of Agricultural and Food Chemistry, v. 53, n. 1, p. 49-56, 2005.

3 CARIBÉ, J.; CAMPOS, J.M. Plantas que ajudam o homem. São Paulo: Cultrix/Pensamentos, 1991. 231 p.

4 CONSTANT, P.B.L.; STRINGHETA, P.C.; SANDI, D. Corantes alimentícios. Boletim do CEPPA, Curitiba, v. 20, n. 2, p. 203-220, 2002.

5 CONSTANT, P.B.L. Extração, caracterização e aplicação de antocianinas de açaí (Euterpre oleralea, M.). 2003. 98 f. Tese (Doutorado em Ciência e Tecnologia de Alimentos), Universidade Federal de Viçosa. Viçosa, 2003.

6 COOPER-DRIVER, G.A. Contributions of Jeffrey Harborne and co-workers to the study of anthocyanins. Phytochemistry, v. 56, n. 3, p. 229-236, 2001.

7 DEBICKY-POSPISIL, J.; LOVRIC, T.; TRINAJSTIC, N.; SABLJIC, A. Anthocyanin degradation in the presence of furfural and 5-hydroxymethylfurfural. Journal of Food Science, v. 48, n. 2, p. 411-416, 1983.

8 DEL POZO-INSFRAN, D.; PERCIVAL, S.S.; TALCOTT, S.T. Açaí (Euterpe oleracea Mart.) polyphenolics in their glycoside and aglycone forms induce apoptosis of HL-60 leukemia cells. Journal of Agricultural and Food Chemistry, $v$. 54, n. 4, p. 1222-1229, 2006.

9 DE ROSSO, V.V.; MERCADANTE, A.Z. The high ascorbic acid content is the main cause of the low stability of anthocyanin extracts from acerola. Food Chemistry, v. 103, n. 3, p. 935-943, 2007.

10 DYRBY, M.; WESTERGAARD, N.; STAPELFELDT, H. Light and heat sensitivity of red cabbage extract in soft drink model systems. Food Chemistry, v. 72, n. 1, p. 431-437, 2001.

11 FALCÃO, L.D.; BARROS, D.M.; GAUCHE, C.; LUIZ, M.T.B. Copigmentação intra e intermolecular de antocianinas: uma revisão. Boletim do CEPPA, Curitiba, v. 21, n. 2, p. 351-366, 2003.

12 FRANCIS, F.J. Analysis of anthocyanins in foods. In: MARKAKIS, P. Anthocyanins as food colors. New York: Academic Press, 1982. p. 181-207.

13 FULEKI, T.; FRANCIS, F. J. Quantitative methods for anthocyanins. 1 Extraction and determination of total anthocyanin in cranberries. Journal of Food Science, v. 33, n.1, p.72-77, 1968.

14 GARZÓN, G.A.; WROLSTAD, R.E. Comparison of the stability of pelargonidin-based anthocyanins in strawberry juice and concentrate. Journal of Food Science, v. 67, n. 4, p. 1288-1299, 2002.

15 HOGAN, S.; CHUNG, H.; ZHANG, L.; JIANRONG, L.; LEE, Y.; DAI, Y.; ZHOU, K. Antiproliferative and antioxidant properties of anthocyanin-rich extracted from açai. Food Chemistry, v. 118, n. 1, p. 208-214, 2010.

16 HUBBERMANN, E.M.; HEINS, A.; STÖCKMANN, H.; SCHWARZ, K. Influence of acids, salt, sugars and hydrocolloids on the color stability of anthocyanin rich black currant and elderberry concentrates. European Food Research and Tecnology, v. 223, n. 1, p. 83-90, 2006.

17 IACOBUCCI, G.A.; SWEENY, J.G. The chemistry of anthocyanins and related flavylium salts. Tetrahedron, v. 39, n. 19, p. 3005-3038, 1983.

18 JURD, L. Some advances in the chemistry of anthocyanins-type plant pigments. In: CHINCHESTER, C.O. (Ed). The chemistry of plant pigments. New York: Academic Press, 1972. p. 123-142.

19 LIMA, V.L.A.G.; MÉLO, E.A.; LIMA, D.E. Efeito da luz e da temperatura de congelamento sobre a estabilidade das antocianinas da pitanga roxa. Ciência e Tecnologia de Alimentos, Campinas, v. 25, n. 1, p. 92-94, 2005.

20 LOPES, T.J.; QUADRI, M.B.; QUADRI, M.G.N. Estudo experimental da adsorção de antocianinas comerciais de repolhoroxo em argilas no processo de batelada. Brazilian Journal of Food Technology,v. 9, n. 1, p. 49-56, 2006.

21 MALIEN-AUBERT, C.; DANGLES, O.; AMIOT, M.J. Color stability of commercial anthocyanin-based extract in relation to phenolic composition. Protective effects by intra- and intermolecular copigmentation. Journal of Agricultural and Food Chemistry, v. 49, n. 1, p. 170-176, 2001. 
22 MOLLOV, P.; MIHALEV, K.; SHIKOV, V.; YONCHEVA, K.; KARAGYOZOV, V. Color stability improvement of strawberry beverage by fortification with polyphenolic copigments naturally occurring in rose petals. Innovative Food Science and Emerging Technologies, v. 8, n. 1, p. 318-321, 2007.

23 MORENO-ALVAREZ, A.J.; MATOS, A.V.; LÓPEZ, E.; BELÉN, D.C. Estabilidad de antocianinas en jugos pasteurizados de mora (Rubus glaucus Benth). Archivos Latinoamericanos de Nutrición, v.52, n. 2, p. 181-186, 2002.

24 MONTES, C.; VICARIO, I.M.; RAYMUNDO, M.; FETT, R.; HEREDIA, F.J. Application of tristimulus colorimetry to optimize the extraction of anthocyanins from Jaboticaba (Myrciaria jaboticaba Berg.). Food Research International, v. 38, n. 8/9, p. 983-988, 2005

25 PERIN, J.D. Efeito de diferentes copigmentos sobre a estabilidade de antocianinas extraídas de berinjela (Solanum melongena L.). 1999. 79 f. Dissertação (Mestrado em Ciência e Tecnologia de Alimentos), Universidade Federal de Viçosa. Viçosa, 1999.

26 POEI-LANGSTON, M.S.; WROLSTAD, R.E. Color degradation in an ascorbic acid-anthocyanin-flavonol model system. Journal of Food Science, v. 46, n. 4, p. 1218-1222, 1981.

27 PROVENZI, G.; FALCÃO, L.D.; FETT R.; LUIZ, M.T.B. Estabilidade de antocianinas de uvas Cabernet Sauvignon com â- e ã-ciclodextrinas. Brazilian Journal of Food Technology, v.9, n.3, p. 165-170, 2006.

28 RABABAH, T.M.; EREIFEJ, K.I.; HOWARD, L. Effect of ascorbic acid and dehydration on concentrations of total phenolics, antioxidant capacity, anthocyanins, and color in fruits. Journal of Agricultural and Food Chemistry, v. 53, n. 1, p. 4444-4447, 2005.

29 RUFINO, M.S.M; ALVES, R.E.; BRITO, E.S.; PÉREZ-JIMÉNEZ, J.; SAURA-CALIXTO, F.D.; MANCINI-FILHO, J. Bioactive compounds and antioxidant capacities of eighteen non-traditional tropical fruits from Brazil. Food Chemistry, v.121, n. 4, p. 996-1002, 2010.

30 SADILOVA, E.; STINTZING, F.C.; KAMMERER, D.R.; CARLE, R. Matrix dependent impact of sugar and ascorbic acid addition on color and anthocyanin stability of black carrot, elderberry and strawberry single strength and from concentrate juices upon thermal treatment. Food Research International, v. 42, n. 1, p. 1023-1033, 2009.

31 SILVA, S.R. Extração e estabilidade de pigmentos antociânicos de frutos de maria-pretinha (Solanum americanum, Mill.). 1996. 65 f. Dissertação (Mestrado em Ciência e Tecnologia de Alimentos), Universidade Federal de Viçosa. Viçosa, 1996.

32 STRINGHETA, P.C. Identificação da estrutura e estudo da estabilidade das antocianinas extraídas da inflorescência de capim gordura (Mellinis minutiflora, Pal de Beauv). 1991. 138 f. Tese (Doutorado em Ciência de Alimentos), Universidade Estadual de Campinas. Campinas, 1991.

33 TEIXEIRA, L.N.; STRINGHETA, P.C.; OLIVEIRA, F.A. Comparação de métodos para quantificação de antocianinas. Revista Ceres, Viçosa, v. 55, n. 4, p. 297-304, 2008.

34 TSAI, P.J.; HSIEH, Y.Y.; HUANG, T.C. Effect of sugar on anthocyanin degradation and water mobility in a roselle anthocyanin model system using ${ }^{17} \mathrm{O}$ NMR. Journal of Agricultural and Food Chemistry, v.52, n. 10, p. 30973099, 2004. 